\title{
Mapping “Broken” Dark Modes Using Cathodoluminescence in a Scanning Electron Microscope
}

\author{
A. C. Y. Liu ${ }^{1,2}$, D. E. Gómez ${ }^{3}$, T. Coenen ${ }^{4}$ \\ 1. Monash Centre for Electron Microscopy, Monash University, Clayton, Australia. \\ 2. School of Physics and Astronomy, Monash University, Clayton, Australia. \\ 3. School of Applied Science, RMIT University, Melbourne, Australia \\ 4. DELMIC BV, Thijsseweg 11, 2629 JA, Delft, The Netherlands
}

Dark plasmon modes are modes that lack a net dipolar character, and hence do not radiate efficiently. They possess a smaller spectral linewidth and longer lifetimes, making them attractive for many applications in sensing and for high-Q cavities [1,2]. A focussed high-energy electron beam can be employed as a local, broad-spectrum excitation source, as the evanescent electric field associated with the fast electrons excites resonances in a polarizable material [3]. The light that is radiated to the farfield can be collected in cathodoluminescence (CL), or the energy loss of the electrons can be measured in electron energy loss spectroscopy (EELS). The spatial resolution of these techniques in the scanning electron microscope (SEM) or scanning/transmission electron microscope (S/TEM) is typically limited by the spatial extent of the evanescent field (order of 10 nm depending on beam energy), making them ideal techniques for mapping the spatial distribution of plasmon modes [4].

The energy lost to a dark mode can be detected in EELS, but since these modes are not radiative, in the strictest sense, these modes cannot be detected in CL [4]. However, due to either inherent or introduced asymmetries in the structure, these dark modes acquire a net dipole resonance and do radiate. We performed hyperspectral CL on gold nanorod trimers fabricated using electron beam lithography [2] using a beam energy of $30 \mathrm{keV}$ and current of 500 pA (FEI Nova Nanosem). The CL was collected using a Delmic SPARC system equipped with an Andor Shamrock 303i spectrometer and Andor iVac spectral camera. We show the concurrent SEM images of a symmetric trimer (Figure 1 a) and a trimer with an introduced asymmetry of an inter-trimer angle of 40 degrees (Figure $1 \mathrm{~b}$ ). The trimers show two resonances, a high-energy symmetric mode and a low-energy mode composed of two degenerate dipole modes [2] (Figure $1 \mathrm{a}$ (ii) and b (ii) showing average spectra corrected for system response). We mapped these modes by mapping the average intensity between 350-500 nm and 600-700 nm, respectively (Figure $1 \mathrm{a}$ (iii-iv) and $\mathrm{b}$ (iii-iv)). While it is clear that the trimer with broken symmetry has a prominent high-energy mode involving all three rods, the symmetric trimer appears to have enough inherent asymmetry to also display this mode. This inherent asymmetry can also be seen in the map of the dipole resonances in Figure 1 a (iv).

Dark modes that are "broken" by asymmetry acquire a dipolar component that allows them to be detected and mapped in CL. Hyperspectral mapping using CL is a powerful method for understanding the effect of nanoscale defects on plasmonic devices [5].

References:

[1] Y-C Chang et al, ACS Nano 6 (2012) p. 3390.

[2] D E Goméz et al, Nano Lett 13 (2013) p. 3722.

[3] T Coenen et al, MRS Bull 40 (2015) p. 359. 
[4] M Kociak and O Stéphan, Chem Soc Rev 43 (2014) p. 3865.

[5] The authors acknowledge the use of facilities in the Monash Centre for Electron Microscopy (ARC LE140100104). We thank Joanne Etheridge, Albert Polman and Tim Davis for many stimulating discussions. This work was performed in part at the Melbourne Centre for Nanofabrication (MCN) in the Victorian Node of the Australian National Fabrication Facility (ANFF). D.E.G. acknowledges the ARC for support through a Future Fellowship (FT140100514) and the ANFF for the MCN Technology Fellowship.

Figure 1. SEM and hyperspectral CL of a symmetric (a) and asymmetric (b) trimer. The average spectra (ii) and the maps generated from mapping the intensity of the features at 350-500 nm (iii) and 600-700 nm (iv) show evidence of both a "broken" dark mode and the radiative dipole modes. The scale bar is $100 \mathrm{~nm}$.
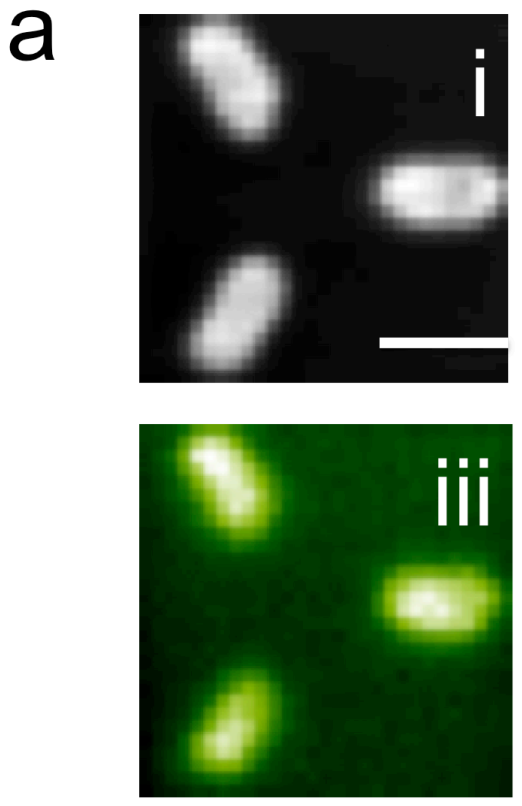

\section{b}
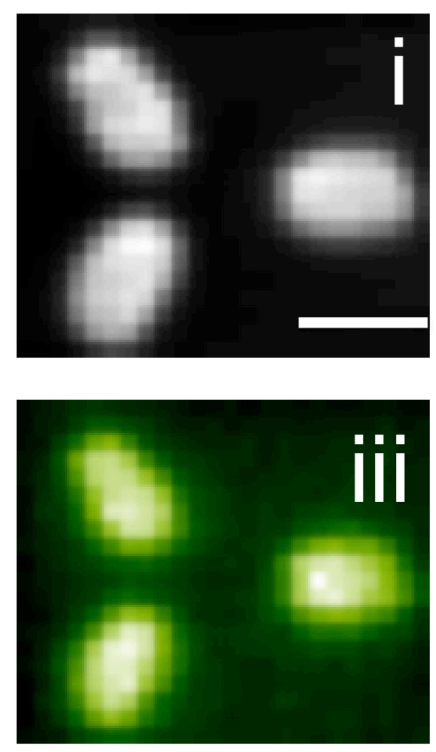
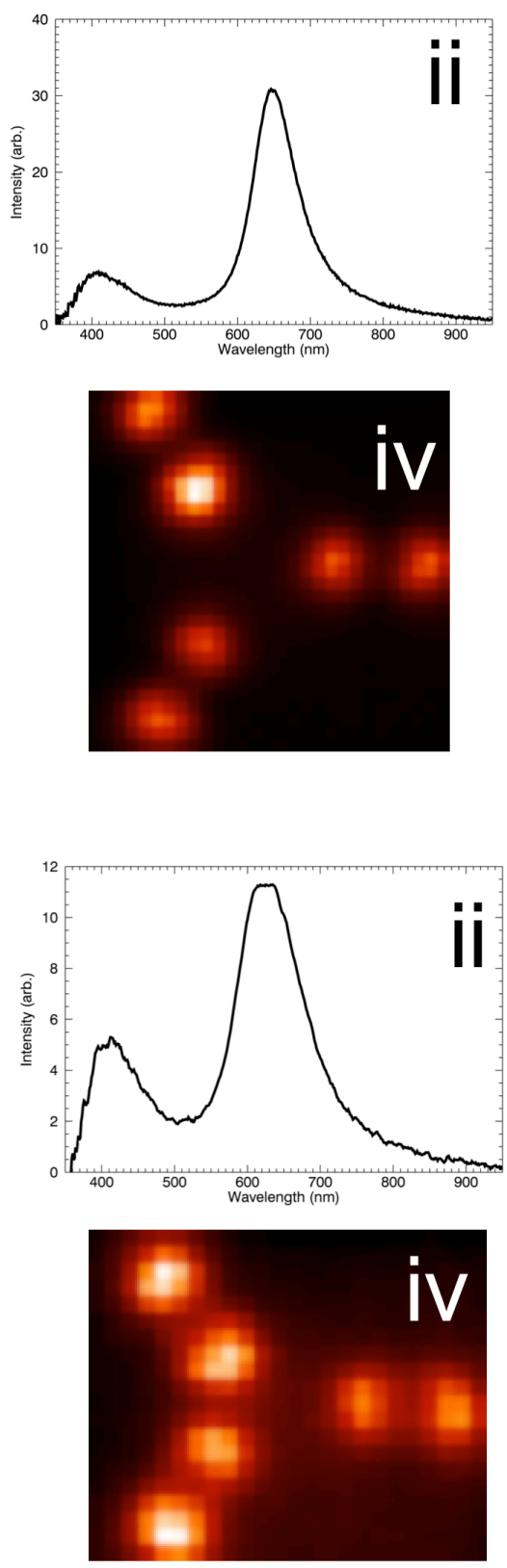